\title{
Zeroes of Chromatic Polynomials: A New Approach to Beraha Conjecture Using Quantum Groups
}

H. Saleur

Service de Physique Théorique ${ }^{\star}$ de Saclay, F-91191 Gif-sur-Yvette Cedex, France

\begin{abstract}
The number of colourings of a graph $G$ with $Q$ or fewer colors is a polynomial in $Q$ known as the chromatic polynomial $P_{G}(Q)$. It coincides with the partition function $\mathscr{Z}_{G}$ of the $Q$ state Potts model on $G$ at zero temperature and in the antiferromagnetic regime $e^{K}=0$. In the planar case, the Beraha conjecture particularizes the numbers $B_{n}=4 \cos ^{2} \frac{\pi}{n}$ as possible accumulation points of real zeroes of $P_{G}$ in the infinite graph limit. We suggest in this work an approach based on recent developments of quantum groups to handle this conjecture. For the square, triangular and honeycomb lattices and systems wrapped on a cylinder $l \times t$, we first exhibit in the $\left(Q, e^{K}\right)$ Potts parameter space a critical line, where $\mathscr{Z}_{G}\left(Q, e^{K}\right)$ has real zeroes converging to and only to the $B_{n}$ 's as $l, t \rightarrow \infty$. The analysis is based on the vertex representation of the $Q$ state Potts model, quantum algebra $U_{q} s l(2)$ properties for $q$ a root of unity, and conformal invariance. $U_{q} s l(2)$ symmetry is present for any $e^{K}$, including the chromatic polynomial case $e^{K}=0$. Using an additional hypothesis on the eigenvalues structure and knowledge of the Potts parameter space, we then argue that for $P_{G}(Q)$, real zeros occur and converge to $B_{n}$ 's, $2 \leqq n \leqq n_{0}$ only, where $n_{0}$ depends on the lattice. Extensions to other kinds of graphs and size dependence of the zeros are discussed. Finally physical applications are also mentioned.
\end{abstract}

\section{Introduction}

Consider a graph $G$, i.e. a set of points and edges joining pairs of points ${ }^{1}$. The number of ways of colouring the points of $G$ with $Q \in \mathbb{N}$ or fewer colours, no two adjacent points having the same colour, is a polynomial in $Q$ known as the chromatic polynomial: $P_{G}(Q)[1]$.

\footnotetext{
* Laboratoire de l'Institut de Recherche Fondamentale du Commissariat à l'Energie Atomique

${ }^{1}$ We suppose that $G$ has no loop, otherwise $P_{G}$ would be trivially 0 for any $Q$. Moreover we can restrict to graphs with no parallel edges, since their presence does not affect the chromatic problem
} 
Besides graph theory, $P_{G}(Q)$ appears also in statistical mechanics[2,3]. If we consider the colours as spins $\sigma=1, \ldots, Q$ of a $Q$ state Potts model such that spins $\sigma_{i}$, $\sigma_{j}$ on adjacent points interact with energy $-J \delta_{\sigma_{i} \sigma_{j}}$ the partition function is

$$
\mathscr{Z}_{G}\left(Q, e^{K}\right)=\sum_{\substack {\{\sigma\} \\
\begin{subarray}{c}{\text { adjacent } \\
\text { spins }{ \{ \sigma \} \\
\begin{subarray} { c } { \text { adjacent } \\
\text { spins } } }\end{subarray}} e^{\beta J \delta_{\sigma_{i} \sigma_{j}}}
$$

where $\beta=\frac{1}{T}, T$ the temperature, $K=\beta J$. Hence in the antiferromagnetic region and for $T \rightarrow 0$ we obtain [3]

$$
P_{G}(Q)=\mathscr{Z}_{G}(Q, 0) \text {. }
$$

Although $P_{G}(Q)$ is defined initially as a number of colourings, we can consider it from now on as a polynomial where $Q$ is indeterminate. Similarly, high temperature expansion $[2,3]$ of $\mathscr{Z}_{G}\left(Q, e^{K}\right)$ allows to define the $Q$-state Potts model for any complex $Q$. One finds

$$
\mathscr{Z}_{G}\left(Q, e^{K}\right)=\sum_{\mathscr{G}}\left(e^{K}-1\right)^{B} Q^{C},
$$

where the sum is over graphs $\mathscr{G}$ containing all sites of $G$ and a subset of its edges. $B$ is the number of edges in $\mathscr{G}, C$ the number of connected components, including isolated sites. Equation (2) holds for complex $Q$ as well.

The zeros of $P_{G}$ are of special interest since for $Q$ integer $P_{G}(Q)=0$ means that the graph $G$ is not $Q$-colorable. We restrict in this paper to planar graphs ${ }^{2}$ (for instance the four colors conjecture [1] means that there should be no graph $G$ such that $\left.P_{G}(4)=0\right)$. Various studies have then revealed the importance [4-9] of the Beraha numbers in the problem

$$
B_{n}=4 \cos ^{2} \frac{\pi}{n},
$$

$B_{1}=4, B_{2}=0, B_{3}=1, B_{4}=2, B_{6}=3$, other $B_{n}$ 's (see Table 1) are non-integer and accumulate in 4 when $n \rightarrow \infty$.

The $B_{n}$ 's are supposed to play a specific role as accumulation points of zeros of chromatic polynomials for large graphs [4-6, 2]. We have found two formulations of the corresponding Beraha conjecture which do not seem fully equivalent. In [6] it is conjectured that among the limits of zeroes of all (not necessarily recursive) families of chromatic polynomials are found all $B_{n}$ 's while a slightly different statement is given in [2], i.e. that the real zeroes of the chromatic polynomial of an arbitrary planar lattice should cluster round limit points belonging to the $B_{n}$ 's when the lattice becomes large. In this paper we consider mainly the second formulation, and hence focus on real zeros. Several numerical checks have been performed [8-10], but besides the case of $B_{5}[7,8]$ and of special recursive maps $[4,6]$ we do not know any exact analysis of the problem.

On the other hand the Beraha numbers (4) occur in several other contexts like the possible values of index for subfactors [11], the classification of unitary conformal field theories [12], or the construction of integrable models [13,14].

\footnotetext{
${ }^{2}$ We recall that a graph $G$ is planar if it can be drawn on the plane in such a way that no two adjacent edges meet except at a common endpoint
} 
Table 1. Numerical values of some Beraha numbers $B_{n}=4 \cos ^{2} \frac{\pi}{n}$

$B_{1}=4$
$B_{2}=0$
$B_{3}=1$
$B_{4}=2$
$B_{5} \simeq 2.618034$
$B_{6}=3$
$B_{7} \simeq 3.2469980$
$B_{8} \simeq 3.414214$
$B_{9} \simeq 3.532089$
$B_{10} \simeq 3.618034$
$\vdots$
$B_{\infty}=4$

Recent developments [14-17] show how the latter subjects are connected due to their common quantum group structure [18]. Our purpose in this paper is to reconsider the colouring problem and Beraha conjecture from the point of view of quantum $s l(2)$. We show that for the square, triangular and honeycomb lattice, there is a line $e^{K}(Q)$ in the Potts model parameter space close to the zero temperature antiferromagnetic line $e^{K}=0$ such that for families of graphs $G$ made of a piece $t \times l$ of lattice wrapped on a cylinder, $\mathscr{Z}_{G}\left(Q, e^{K}\right)$ has real zeros converging to all the $B_{n}$ 's in the thermodynamic limit $t, l \rightarrow \infty$. The properties of the Potts model are singular at these numbers. For the chromatic polynomial $\mathscr{Z}_{G}(Q, 0)$ itself, we show under some additional assumptions that real zeros converge to a subset $B_{n}, n \leqq n_{0}$ where $n_{0}$ depends on the lattice ( $n_{0}=6$ for the square lattice, 14 for the triangular, 4 for the honeycomb). We discuss possible extensions of our results to other two dimensional graphs. Physical applications are also mentioned.

\section{Square Lattice-General Description}

We start with the square lattice and consider a $Q$ (integer)-state Potts model on the planar graph $G_{l, t}$ of Fig. 1, with free boundary conditions (BC) on the top and bottom row, and periodic $B C$ in the time direction. We denote the horizontal (vertical) coupling $J_{1}\left(J_{2}\right)$. The transfer matrix $\tau_{\text {Potts }}$ from one column to the next is expressed as a product of bond matrices $X$ that add some horizontal or vertical edge. Defining

we have

$$
\begin{aligned}
\left(e_{2 i-1}\right)_{\sigma \sigma^{\prime}} & =Q^{-1 / 2} \prod_{\substack{j=1 \\
j \neq i}}^{l} \delta_{\sigma_{j} \sigma_{j}^{\prime}}, \\
\left(e_{2 i}\right)_{\sigma \sigma^{\prime}} & =Q^{1 / 2} \delta_{\sigma_{i} \sigma_{i+1}} \prod_{j=1}^{l} \delta_{\sigma_{j} \sigma_{j}^{\prime}},
\end{aligned}
$$

where

$$
X_{2 i-1}=\left(x_{1}+e_{2 i-1}\right), \quad X_{2 i}=\left(1+x_{2} e_{2 i}\right),
$$

$$
x=Q^{-1 / 2}\left(e^{K}-1\right)
$$




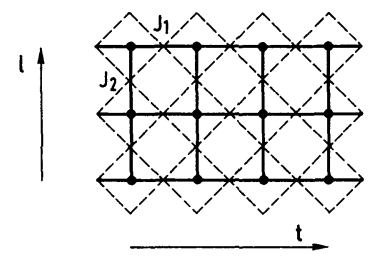

Fig. 1. Square lattice graph $G_{l, t}$, and its medial graph $G^{\prime}$. Opposite sides in the time direction $t$ are identified

and

$$
\tau_{\text {Potts }}=Q^{l / 2} \prod_{i=1}^{l} X_{2 i-1} \prod_{i=1}^{l-1} X_{2 i} .
$$

The operators $e$ satisfy the Temperley Lieb projector algebra

$$
\left\{\begin{aligned}
e_{i}^{2} & =Q^{1 / 2} e_{i} \\
e_{i} e_{i \pm 1} e_{i} & =e_{i} \\
{\left[e_{i}, e_{j}\right] } & =0 \quad|i-j| \geqq 2
\end{aligned}\right.
$$

and the partition function is

$$
\mathscr{Z}_{G}\left(Q, e^{K_{1}}, e^{K_{2}}\right)=\operatorname{Tr}\left(\tau_{\text {Potts }}\right)^{t} .
$$

An important step in our approach will consist in using the other representation of algebra (8) provided by the staggered 6-vertex model. We introduce vertices on the medial graph $G^{\prime}$, with weights

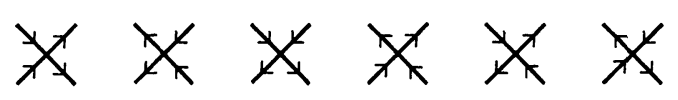

$$
\begin{aligned}
& \text { or } \begin{array}{cccccc}
x_{1} & x_{1} & -1 & -1 & x_{1}+q^{-1} & x_{1}+q \\
1 & 1 & -x_{2} & -x_{2} & 1+x_{2} q^{-1} & 1+x_{2} q
\end{array}
\end{aligned}
$$

depending on whether the vertex stands on an horizontal or vertical bond of $G$. Matrices which add a vertex are

$$
X_{2 i-1}=\left(x_{1}+e_{2 i-1}\right), \quad X_{2 i}=\left(1+x_{2} e_{2 i}\right),
$$

where $e$ acts in the tensor product of $2 l$ spin $\frac{1}{2}$ representations $\mathscr{H}=\varrho_{1 / 2}^{\otimes 2 l}$ as

$$
e_{i}=\mathbb{1} \otimes \mathbb{1} \otimes \ldots \otimes\left(\begin{array}{cccc}
0 & 0 & 0 & 0 \\
0 & q^{-1} & -1 & 0 \\
0 & -1 & q & 0 \\
0 & 0 & 0 & 0
\end{array}\right) \otimes \mathbb{1} \ldots
$$

where

$$
Q^{1 / 2}=q+q^{-1}
$$


It was then noticed in [18] that (12), (11) and hence the whole vertex transfer matrix $\tau_{\text {vertex }}$ commute with the quantum algebra $U_{q} s l(2)$. The latter is a deformation of the universal enveloping algebra of $s l(2)$ with generators $S^{ \pm}, S^{Z}$ obeying

$$
\left\{\begin{array}{l}
{\left[S^{+}, S^{-}\right]=\frac{q^{2 S^{Z}}-q^{-2 S^{Z}}}{q-q^{-1}}} \\
{\left[S^{Z}, S^{ \pm}\right]= \pm S^{ \pm}}
\end{array}\right.
$$

Acting in $\mathscr{H}$ we have

$$
\begin{aligned}
S^{Z} & =\sum_{i=1}^{2 l} \sigma_{i}^{Z}, \\
S^{ \pm} & =\sum_{i=1}^{2 l} q^{\sigma_{1}^{Z}} \otimes \ldots \otimes q^{\sigma_{i-1}^{Z}} \otimes \sigma^{ \pm} \otimes q^{-\sigma_{i+1}^{Z}} \otimes \ldots \otimes q^{-\sigma_{2 t}^{Z}},
\end{aligned}
$$

where $\sigma$ are usual $s l(2)$ generators acting in $\varrho_{1 / 2}$. The relations (8) define the commutant of $U_{q} s l(2)$ in $\mathscr{H}$.

In the $q$ generic case, the representations of $U_{q} s l(2)$ are in one to one correspondence with $\operatorname{sl}(2)(q=1)$ case. We label them as $\varrho_{j}$, with dimension $2 j+1$. Due to

$$
\left[\tau_{\text {vertex }}, U_{q} s l(2)\right]=0
$$

we can decompose the space $\mathscr{H}$ onto eigenspaces as

where

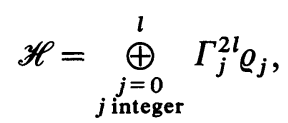

$$
\Gamma_{j}^{2 l}=\left(\begin{array}{c}
2 l \\
l-j
\end{array}\right)-\left(\begin{array}{c}
2 l \\
l-j-1
\end{array}\right)
$$

Associated to spin $j$ we have eigenvalues $\lambda_{j}^{\alpha}, \alpha=1, \ldots, \Gamma_{j}^{2 l}$ (in increasing order).

$e$ matrices $(4,12)$ satisfying the same algebra, $\tau_{\text {Potts }}$ and $\tau_{\text {vertex }}$ have some eigenvalues in common. Their precise correspondence is worked out using for instance graphical arguments. On $G$, the partition function of the $Q$ (integer)- state Potts model is $[2,3]$

$$
\mathscr{Z}_{G}\left(Q, e^{K_{1}}, e^{K_{2}}\right)=\sum_{\mathscr{G}}\left(e^{K_{1}}-1\right)^{B_{1}}\left(e^{K_{2}}-1\right)^{B_{2}} Q^{C},
$$

which now makes sense for any real $Q$. The graphs $\mathscr{G}$ are in one to one correspondence with polygon decomposition $\mathscr{G}^{\prime}$ of $G^{\prime}$ [19], and one finds using Euler's relation

$$
\mathscr{Z}_{G}\left(Q, e^{K_{1}}, e^{K_{2}}\right)=Q^{l t / 2} \sum_{\mathscr{G}^{\prime}}\left(x_{1}\right)^{B_{1}}\left(x_{2}\right)^{B_{2}} Q^{P / 2},
$$

where $P$ is the number of polygons in $\mathscr{G}^{\prime}$.

The polygons can be oriented, and their weight $Q^{1 / 2}$ turned into a local form by associating complex weights $q^{ \pm 1 / 4}$ to each left or right turn. Attaching oriented polygons at each site of the medial lattice reproduces the 6-vertex model with 
weights (10) (the additional minus sign is introduced there for convenience and does not change the resulting partition function). A special case must be taken to deal with the periodic BC $[19,21]$, and one finds

$$
\mathscr{Z}_{G}\left(Q, e^{K_{1}}, e^{K_{2}}\right)=Q^{l t / 2} \sum_{\substack{j=0 \\ j \text { integer }}}^{l}(2 j+1)_{q} \sum_{\alpha=1}^{\Gamma_{j}^{2 l}}\left(\lambda_{j}^{\alpha}\right)^{t},
$$

where we have introduced the $q$ analog of the dimension of representations $\varrho_{j}$,

$$
(y)_{q}=\frac{q^{y}-q^{-y}}{q-q^{-1}}
$$

(for simplicity, the $l$ dependence of eigenvalues is not indicated in our notation). Formula (20) holds true for any real $Q$. When $Q$ is not integer the coefficients in (20) can be non-integer, reflecting the fact that the partition function of the geometrical model $(18,19)$ is not the trace of some transfer matrix raised to the $t$.

\section{Representations of $U_{q} s l(2)$ when $Q$ is a Root of Unity}

The representation theory of $U_{q} s l(2)$ is qualitatively very different $[15,22,23]$ from the generic case when $q$ is a root of unity $q=e^{i \pi m / n}$. As shown in [15], sets of states which for $q$ generic belong to representations $\varrho_{j}, \varrho_{j^{\prime}}$ can gather in a larger (Type I) representation $\varrho_{j j^{\prime}}$ which is indecomposable but not irreducible when

$$
\begin{gathered}
j^{\prime}=-1-j \bmod n \\
0 \leqq j^{\prime}-j \leqq n-1
\end{gathered}
$$

This pairing occurs in a maximal way. For a given $0 \leqq j<\frac{n-1}{2}$ we have spins $0 \leqq j<n-1-j=j_{1}<j+n=j_{2}<\ldots<j_{k} \leqq 2 l$ such that $\left(j_{i}, j_{i+1}\right)$ satisfy (21). Then all $\Gamma_{j_{k}}^{2 l}$ representations $\varrho_{j_{k}}$ are paired with a subset $\Gamma_{j_{k}}^{2 l}$ of the $\Gamma_{j_{k-1}}^{2 l}$ representations $\varrho_{j_{k-1}}$. The remaining $\varrho_{j_{k-1}}$ are paired with a subset $\Gamma_{j_{k-1}}^{2 l}-\Gamma_{j_{k}}^{2 l}$ of the $\Gamma_{j_{k-2}}^{2 l}$ representations $\varrho_{j_{k-2}}$, etc.

In this way all $j_{i}, i \geqq 1$ representations are paired. We are left with a number

$$
\Omega_{j}^{2 l}=\Gamma_{j}^{2 l}-\Gamma_{j_{1}}^{2 l}+\Gamma_{j_{2}}^{2 l}-\Gamma_{j_{3}}^{2 l}+\ldots+(-)^{k} \Gamma_{j_{k}}^{2 l}
$$

of representations which cannot be paired. They are called of Type II, and have still the same structure as $\operatorname{sl}(2)$ ones. The case $j=\frac{n-1}{2}$ is treated separately since it is invariant under (21). Representations with spins $j=\frac{n-1}{2} \bmod n$ are not paired, and also in correspondence with $\operatorname{sl}(2)$ ones. They are nevertheless called of Type $I$. Hence, because $(2 j+1)_{q}$ changes sign in (21), Type I representations are characterized by their $q$ dimension being zero.

The transfer matrix of the vertex model $\tau_{\text {vertex }}$ commutes with $U_{q} s l(2)$, hence when two representations $\varrho_{j}, \varrho_{j^{\prime}}$ get paired, the corresponding eigenvalues $\lambda_{j}, \lambda_{j^{\prime}}$ which were not related for $q$ generic become identical. The expression (22) thus makes sense for contributions to the partition function also, and (20) reads then

$$
\mathscr{Z}_{G}\left(Q, K_{1}, K_{2}\right)=Q^{l t / 2} \sum_{j=0}^{E(n / 2)-1}(2 j+1)_{q} \chi_{j}^{(l, t)}
$$


where $E(y)$ is the integer part of $y$, and

$$
\chi_{j}^{(l, t)}=\sum_{\alpha=1}^{\Gamma_{j}^{2 l}}\left(\lambda_{j}^{\alpha}\right)^{t}-\sum_{\alpha=1}^{\Gamma_{j_{1}}^{2 l}}\left(\lambda_{j_{1}}^{\alpha}\right)^{t}+\ldots+(-)^{k} \sum_{\alpha=1}^{\Gamma_{j_{k}}^{2 l}}\left(\lambda_{j_{k}}^{\alpha}\right)^{t} .
$$

In the latter expression the $\lambda_{j}^{\alpha}$ are defined by continuity from the irrational case.

\section{The "Non-Physical" Self Dual Line: Conformal Properties}

So far our discussion did not depend on the values of the couplings and actually may be generalized to an inhomogeneous Potts model. To proceed further we need, besides the $U_{q} s l(2)$ structure, some information on the eigenvalues $\lambda$ themselves. Unfortunately, not much is known for general $K_{1}, K_{2}$. The most favorable case is the critical self dual [3] line characterized by

$$
x_{1} x_{2}=1 \text {. }
$$

In this case the staggered 6-vertex model becomes homogeneous [1]. Moreover the weights of two last kind of vertices can be made equal by a gauge transformation, giving a zero field 6-vertex model. This is known to be (partly) solvable. In the isotropic $x_{1}=x_{2}$ case, $(25)$ becomes $x^{2}=1$. In Refs. $[15,21]$ the case $x_{1}, x_{2}>0$ was considered, corresponding to the ferromagnetic critical Potts model. There it was shown how the peculiarities of $U_{q} s l(2)$ representations for $q$ a root of unity were related to the existence of minimal conformal theories in the continuum limit, the degeneracies in the spectrum of $\tau_{\text {vertex }}$ corresponding to the appearance of null states in Virasoro modules [24]. This however was a subtle effect affecting small eigenvalues, and not manifest directly in the physical properties of the $Q=4 \cos ^{2} \frac{\pi m}{n}$ Potts model. For instance the largest eigenvalue of $\tau_{\text {vertex }}$ appears in the $j=0$ sector, and the associated representation never gets mixed. Hence for $x=1$ in particular the free energy of a Potts model on an infinite cylinder is regular for any $Q$.

We consider here the other case $x_{1}, x_{2}<0$. In the isotropic case, $e^{K}=1-Q^{1 / 2}$, which is not very physical since $K$ is complex (with imaginary part equal to $\pi$ ) for $Q>1$. However it should be of some interest since the $B$ dependent term in (3) is negative, as for the chromatic polynomial case. We have

$$
\mathscr{Z}_{G}(Q, x=-1)=\sum_{\mathscr{G}}\left(-Q^{1 / 2}\right)^{B} Q^{C},
$$

thus $\mathscr{Z}_{G}$ on the line $x=-1$ is a polynomial in $Q^{1 / 2}$.

With the geometry we choose, $\tau_{\text {vertex }}$ does not seem to be directly diagonalizable using the Bethe ansatz [25]. We can however consider the line (25) with $x_{1}, x_{2}$ negative and take the limit $x_{2} \rightarrow 0^{-}, x_{1} \rightarrow-\infty$ to deal instead with the hamiltonian problem

$$
H=\frac{-2(\pi-\gamma)}{\pi \sin \gamma} \sum_{i=1}^{2 l-1} \sigma_{i}^{X} \sigma_{i+1}^{X}+\sigma_{i}^{Y} \sigma_{i+1}^{Y}+\cos \gamma \sigma_{i}^{Z} \sigma_{i+1}^{Z}+\frac{i}{2} \sin \gamma\left(\sigma_{1}^{Z}-\sigma_{2 l}^{Z}\right),
$$

where $q=e^{i \gamma}, 0 \leqq \gamma \leqq \frac{\pi}{2}$. Bethe ansatz equations can be written for (27). As shown in [15], associated Bethe states with $S^{z} \geqq 0$ are annihilated by the raising operator $S^{+}$. 
For $q$ generic the Bethe solutions are believed to give all the eigenenergies $\varepsilon_{j}^{\alpha}$, while their multiplicity $2 j+1$ follows from quantum group symmetry [26].

For each $0 \leqq j \leqq l$, it is possible to study the smallest $\varepsilon_{j}^{\alpha}=\varepsilon_{j}^{1}$. Under the usual real roots assumption [27] one finds, where $\varepsilon$ is the non-universal extensive part of the energy

$$
\lim _{l \rightarrow \infty} \frac{2 l}{\pi}\left(\varepsilon_{j}^{1}-\varepsilon\right)=h_{j}-\frac{c}{24}
$$

with central charge

$$
c=1-6 \frac{(\delta-1)^{2}}{\delta}
$$

conformal weights [28]

$$
h_{j}=\frac{j(j-\delta+1)}{\delta}
$$

and we have introduced

$$
\delta=\frac{\pi}{\gamma}
$$

The Kac formula in parametrization (29) reads

$$
h_{r s}=\frac{(\delta r-s)^{2}-(\delta-1)^{2}}{4 \delta}
$$

and $h_{j}=h_{1,1+2 j}$. Although results (28-30) are established in the $l \rightarrow \infty$ limit, corrections to scaling analysis [17] suggests that the relative position of levels $\varepsilon_{j}^{1}$ described from (30) holds true - up to any fixed energy - for $l$ large enough. In fact the numerical study shows this order is correct for any finite $l$ already. We see from (30) that the minimum $h_{j}$ occurs for spin

$$
J=\left[\frac{\delta-1}{2}\right]
$$

where $[y]$ means the integer closer to $y$ (ambiguity for $\delta=2 n^{\prime}$ is discussed later). Due to $U_{q} s l(2)$ symmetry, all levels $\varepsilon_{j}^{1}$ are repeated $2 j+1$ times. Some of the $h_{j}$ 's are negative, in connection with the non-unitarity of the theory. The apparent central charge, i.e. the one observed by measuring the ground state $\varepsilon_{J}^{1}$, is

$$
c_{\text {app }}=c-24 h_{J} \text {. }
$$

Besides the $\varepsilon_{j}^{1}$, not much can be calculated exactly. But Bethe ansatz and conformal invariance arguments suggest that

$$
\lim _{l \rightarrow \infty} K_{j}^{(l)}=\lim _{l \rightarrow \infty} \sum_{\alpha=1}^{r_{j}^{2 l}} y^{2 l / \pi\left(\varepsilon_{j}^{\alpha}-\varepsilon\right)}=\frac{y^{h_{j}}-y^{h_{-1}-j}}{P(y)}=K_{j}(y),
$$

where $y$ is an undeterminate variable in the generating function and $P(y)$ $=\prod_{1}^{\infty}\left(1-y^{n}\right)$. 
Table 2. a) Energies of the $X X Z$ hamiltonian (27) at $l=2, \delta=\sqrt{7}$. b) Scaled gaps are indicated, to be compared with their asymptotic (conformal weights) values

$$
\begin{aligned}
& \text { a) } \\
& \mathbf{S}=\mathbf{0} \\
& -0.824208 ; \quad \varepsilon_{0}^{1}=-0.606536 ; \quad-0.375083 ; \quad 0.125028 ; \quad 1.074263 ; \quad \varepsilon_{0}^{2}=1.356701 \\
& \mathbf{S}=1 \\
& \varepsilon_{1}^{1}=-0.824208 ; \quad-0.375083 ; \quad \varepsilon_{1}^{2}=0.125028 ; \quad \varepsilon_{1}^{3}=1.074263 \\
& \mathbf{S}=2 \\
& \varepsilon_{2}^{1}=-0.375083 \\
& \text { b) } \\
& \frac{2 l}{\pi}\left(\varepsilon_{0}^{2}-\varepsilon_{0}^{1}\right)=2.4997 \\
& \frac{2 l}{\pi}\left(\varepsilon_{1}^{1}-\varepsilon_{0}^{1}\right)=-0.27715 \quad h_{1} \simeq-0.21653 \\
& \frac{2 l}{\pi}\left(\varepsilon_{1}^{2}-\varepsilon_{1}^{1}\right)=1.2086 \quad 1 \\
& \frac{2 l}{\pi}\left(\varepsilon_{1}^{3}-\varepsilon_{1}^{1}\right)=2.4172 \quad 2 \\
& \frac{2 l}{\pi}\left(\varepsilon_{2}^{1}-\varepsilon_{0}^{1}\right)=0.29469 \quad h_{2} \simeq 0.26779
\end{aligned}
$$

From $(30,32)$ one deduces easily the structure of the whole spectrum. As an example we consider $\delta=\sqrt{7}, l=2$. From (33) the ground state occurs for $J=1, \varepsilon_{1}^{1}$. The next level is $\varepsilon_{0}^{1}$, then $\varepsilon_{2}^{1}$ appear after $\varepsilon_{1}^{2}, \varepsilon_{1}^{3}$ and finally $\varepsilon_{0}^{2}$. Numerical values are given Table 2, together with the asymptotic expected ones.

Concerning the full vertex model, we found numerically a completely similar structure. If we set

$$
x_{2}=\frac{\sin u}{\sin (\gamma-u)}, \quad \gamma<u<\pi(\bmod \pi)
$$

the anisotropy factor is

$$
\text { anisotropy }=\operatorname{tg}\left[\frac{\pi^{2}-\pi u}{2(\pi-\gamma)}\right]
$$

equal to 1 for $x_{2}=-1, u=\frac{\gamma}{2}+\frac{\pi}{2}$. It is also possible to consider the Potts model in the slightly different geometry of [25] where Bethe ansatz can be applied for any $u$. The results are similar, and for clarity we do not discuss them.

\section{Singularities of the Potts Model}

The main result of the last section is that the value of $j$ at which the ground state occurs moves with $\delta$. This is very different from the usual $x=1$ case, and combined with (23) plus $U_{q} s l(2)$ representation theory, will lead to some singularities for the Potts model. 
We suppose $l$ large enough such that in some fixed interval of $\delta$, the two largest eigenvalues of $\tau_{\text {vertex }}$ occur at spin expected from (33). Note that if the largest eigenvalue $\Lambda=\lambda_{J}^{1}$, the next one $\Lambda^{\prime}$ is either $\lambda_{J+1}^{1}$ or $\lambda_{J-1}^{1}$ depending on whether $\frac{\delta-1}{2} \in[J, J+1]$ or $[J-1, J]$. For $\delta$ irrational, there is no possible cancellation in the coefficients of (23). The ground state $\lambda_{J}^{1}$ of the vertex model is also the one of the Potts model, and in the infinite strip limit $t \rightarrow \infty^{3}$,

$$
f_{\text {Potts }}^{l}=f_{\text {vertex }}^{l}=\frac{1}{2 l} \ln \lambda_{J}^{1} .
$$

When $\delta$ is a rational, additional degeneracies occur among the $\lambda$ 's and accordingly cancellations in (23). If $\delta$ is not integer, $\delta=\frac{n}{m},(2 J+1)_{q}$ is ${ }^{4}$ non-zero due to $m>1$. Moreover the representation $\varrho_{J}$ attached to $\lambda_{J}^{1}$ cannot mix into a Type I. For, if that were the case, $\lambda_{J}^{1}$ would coincide with some $\lambda_{n-1-J \bmod n}^{\alpha}$. But the next eigenvalue $\Lambda^{\prime}$ occurs for a highest weight at $J \pm 1$, and none of the $\varrho_{J \pm 1}$ representations mixes with $\varrho_{J}$ for $m>1$. Hence (35) still holds true when $\delta$ is rational $\in Q-\mathbb{N}$.

Suppose now $\delta$ integer $=n$. We have to distinguish between odd and even case. If $n=2 n^{\prime}+1, J=[n]=n^{\prime} . \varrho_{J}$ is one of these representations which are still isomorphic to $\operatorname{sl}(2)$ ones, but with $q$ dimension zero $(2 J+1)_{q}=0$. Hence the contribution of $\lambda_{J}^{1}$ suddenly disappears from (20). If $n=2 n^{\prime},[J]$ is right in the middle of $n^{\prime}$ and $n^{\prime}-1$. From (30), $h_{n^{\prime}}=h_{n^{\prime}-1}$, thus $\lambda_{n^{\prime}}^{1}$ and $\lambda_{n^{\prime}-1}^{1}$ are asymptotically degenerate. In fact, due to $U_{q} s l(2)$ symmetry and since $n^{\prime}, n^{\prime}-1$ obey (22), they are equal for finite $l$, the representations $\varrho_{n^{\prime}}$ and $\varrho_{n^{\prime}-1}$ mixing into a Type I. The $q$-dimension of $\varrho_{n^{\prime}, n^{\prime}-1}$ being zero, the contribution of $\lambda_{J}^{1}$ again disappears from (20).

We do not expect any additional degeneracies in the spectrum, such that $\lambda_{J}^{1}$ would become equal to still another $\lambda_{j}^{\alpha}$ at $\delta=n$, just replacing the missing contribution of $\lambda_{J}^{1}$ in (20). This can be fully established using the conformal invariance description (34). Indeed, we expect in the continuum limit the eigenvalues $\lambda$ to scale as $\lambda \simeq \Lambda e^{\frac{-\pi h}{l}}$, where $h$ is some conformal weight. Hence, each term in the summation (24) reads asymptotically

$$
\lim _{t, l \rightarrow \infty} \sum_{\alpha=1}^{\Gamma_{j}^{2 l}}\left(\lambda_{j}^{\alpha}\right)^{t} \simeq \Lambda^{t} e^{h_{J} \pi t / l} K_{j}\left(e^{-\pi t / l}\right)
$$

\footnotetext{
${ }^{3}$ In Def. (35), $f_{\text {Potts }}^{l}$ is the free energy per bond of the Potts model, equal to half of the free energy per ${ }^{4} \delta=\frac{n}{m}, n$ and $m$ coprimes. $m \geqq 2$ and $\delta \geqq 2$ imply $n \geqq 4$. Then $J=\left[\frac{\delta-1}{2}\right]=\left[\frac{n / m-1}{2}\right]$ $\leqq\left[\frac{n-2}{4}\right]=$ either $E\left(\frac{n-2}{4}\right)$ or $E\left(\frac{n-2}{4}\right)+1$. Then $J<\frac{n-2}{4}+1=\frac{n}{4}+\frac{1}{2}$ and $1 \leqq 2 J+1$ $<\frac{n}{2}+2 \leqq n$. Moreover, if $2 J+1$ divides $n, 2 J+1=\frac{n}{p}, p>1, p$ does not divide $m$ for by hypothesis $n$ and $m$ are coprimes. Hence $\sin \pi(2 J+1) \frac{m}{n} \neq 0$
} 
Table 3. Logarithms of $\tau_{\text {vertex }}$ eigenvalues for $\delta=n=4, u=\pi-\frac{\gamma}{2}=7 \frac{\pi}{8}, X=1+\frac{\sin u}{\sin (\gamma-u)} e, l=3$. $\frac{n-1}{2}=\frac{3}{2}$, thus $J$ is 1 or $2, \varrho_{1}$ and $\varrho_{2}$ are mixed. We underlined levels of highest weight states kept in $\tau_{\text {Potts. }}$. The ground state of $\tau_{\text {Potts }}$ is the $\sigma^{\text {th }}$ excited state of $\tau_{\text {vertex }}$

$\mathbf{S}=\mathbf{0}$

$0.18248 \times 2 ; \quad 0 \times 2 ; \quad-0.24840 \times 2 ; \quad-0.61799 ; \quad-0.881374 \times 2 ; \quad-1.036641 ;$

$-1.51439 \times 2 ; \quad-1.76274 ; \quad-1.95022 \times 2 ; \quad-2.18139 ; \quad-2.22547$;

$-2.644121 ; \quad-\frac{1.76274 ;}{3.370226 ;}-3.78887$

$\mathrm{S}=1$

$0.18248 \times 2 ; \quad 0 ; \quad-0.24840 \times 2 ; \quad-0.61799 ; \quad-0.881374 \times 2 ; \quad-1.51439 \times 2 ;$

$-1.95022 \times 2 ; \quad-2.181397 ; \quad-2.644121 ; \quad-3.370226$

$\mathbf{S}=2$

$0.18248 ; \quad 0 ; \quad-0.24840 ; \quad-0.881374 ; \quad-1.51439 ; \quad-1.94522$

$\mathbf{S}=3$

0

A naive calculation of $\lim _{t, l \rightarrow \infty} \chi_{j}^{(l, t)}$ however breaks down (this again is very different from the $x=1$ case discussed in [15]). Indeed in the infinite sum

$$
\chi_{j}=K_{j}(y)-K_{n-1-j}(y)+K_{j+n}(y)-K_{n-1-j+n}(y)+\ldots
$$

the contribution of $y^{h}$ cancel due to

$$
h_{j}=h_{n-1-j} \text {. }
$$

Hence $\chi_{j} \rightarrow 0$ while $\Lambda^{t}$ diverges. This phenomenon is similar to the one encountered by Andrews et al. [13] in their calculation of local height probabilities in regime II. It means that besides the above cancellation of largest eigenvalues, many other ones occur, and that the first eigenvalue contributing to the Potts partition function (24) is very far in the vertex spectrum. A numerical example is shown in Table 3.

\section{Zeros of the Potts Model Partition Function on the Self Dual Line}

Associated to the above mechanisms are zeros of $\mathscr{Z}_{G}(Q, x=-1)$. Indeed consider first the case $\delta \simeq n=2 n^{\prime}+1$. Expand $\mathscr{Z}_{G}$ on the vertex-Potts eigenvalues as in (20),

$$
\mathscr{Z}_{G}(Q)=Q^{l t / 2} \sum_{\substack{j=0 \\ j \text { integer }}}^{l}(2 j+1)_{q} \sum_{\alpha=1}^{\Gamma_{j}^{2 l}}\left(\lambda_{j}^{\alpha}\right)^{t} .
$$

$\mathscr{Z}_{G}=0$ can be rewritten as

$$
(2 J+1)_{q}=-\sum_{\alpha=2}^{\Gamma_{J}^{2 l}}(2 J+1)_{q}\left(\frac{\lambda_{J}^{\alpha}}{\lambda_{J}^{1}}\right)^{t}-\sum_{\substack{j=0 \\ j \neq J}}^{l}(2 j+1)_{q} \sum_{\alpha=1}^{\Gamma_{j}^{2 l}}\left(\frac{\lambda_{j}^{\alpha}}{\lambda_{J}^{1}}\right)^{t} .
$$

In the $t \rightarrow \infty$ limit, because $\lambda_{J}^{1}$ is non-degenerate, the right member goes to zero, and the solution of (40) in variable $Q$ is $B_{n}$. For $t$ finite, we can expand both sides 
which are analytic in $Q$ around $Q=B_{n}$. The coefficients are real since eigenvalues $\lambda$ are real or appearing in pairs of complex conjugate. Hence (40) is solved by real numbers $Q(t)$ which converge exponentially fast towards $B_{n}$ for $t \rightarrow \infty$. For $\delta \simeq n$ $=2 n^{\prime}$, a similar result is reached taking into account the multiplicity 2 (with opposite prefactors) of the largest eigenvalue $\lambda_{n^{\prime}}^{1}=\lambda_{n^{\prime}-1}^{1}$ for $\delta=2 n$.

Hence we expect real zeroes converging to all the $B_{n}$ 's, for $l \gtrsim \frac{n-1}{2}$ and $t \rightarrow \infty$.

We can also consider both $l, t$ increasing simultaneously. For $l \gtrsim \frac{n-1}{2}$, the largest eigenvalue occurs at $J$. Due to the conformal invariance result on the behaviour of other $\lambda$ 's, the sums in (40) converge for any $t$ when $l \rightarrow \infty$, and can be expanded as before around $Q=B_{n}$ for $t$ large.

Besides the $B_{n}$, no other singularity shows up in the analysis, especially for $Q=4 \cos ^{2} \frac{\pi m}{n}, m>1$. These are thus the only accumulation points of real zeros (we do not have access here to information on the pattern of complex zeroes).

\section{Non-Physical Critical Lines for the Triangular and Honeycomb Lattices}

It is important to consider also some other lattices, as the Beraha conjecture we want to address applies irrespectively of the graph $G$.

We introduce the $Q$-state Potts model on the triangular lattice (Fig. 2) $G$ with three couplings $J_{1} J_{2} J_{3}$ depending on the type of bond. With the same definitions as in $(5,6)$ we have

$$
\left\{\begin{array}{rlrl}
X_{2 i-1} & =\left(x_{1}+e_{2 i-1}\right) & & \\
X_{4 i+2} & =\left(1+x_{2} e_{4 i+2}\right), & \tilde{X}_{4 i+2} & =\left(1+x_{3} e_{4 i+2}\right) \\
X_{4 i} & =\left(1+x_{3} e_{4 i}\right), & \tilde{X}_{4 i}=\left(1+x_{2} e_{4 i}\right),
\end{array}\right.
$$

a)

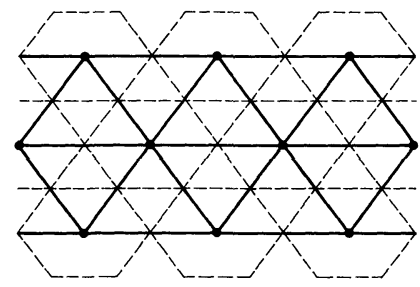

b)

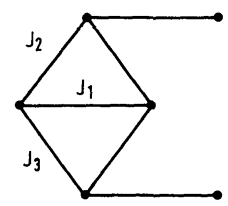

Fig. 2. a) The triangular lattice and its medial Kagomé lattice. b) The column to column transfer matrix for this example reads

$$
\tau_{\text {Potts }}=Q^{3 / 2} X_{5} X_{1} \tilde{X}_{4} \tilde{X}_{2} X_{3} X_{4} X_{2}
$$


and for $l_{\text {odd }}=2 l^{\prime}+1$,

$$
\tau_{\mathrm{Potts}}=Q^{l / 2} \prod_{i=0}^{l^{\prime}} X_{4 i+1} \prod_{i=1}^{l-1} \tilde{X}_{2 i} \prod_{i=1}^{l^{\prime}} X_{4 i-1} \prod_{i=1}^{l-1} X_{2 i}
$$

The important thing is that the Temperley Lieb algebra (8) is still hidden in the Potts transfer matrix. Hence, as in the square lattice case, we can use the other (vertex) representation. The medial lattice $G^{\prime}$ is the Kagomé lattice. Three types of vertices occur with the following weights:

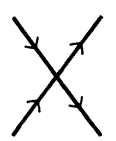

$x_{1}$

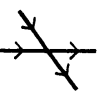

1

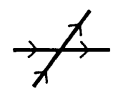

1

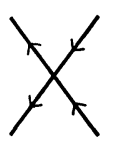

$x_{1}$

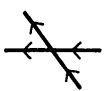

1

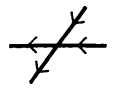

1

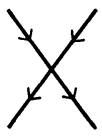

$-1$
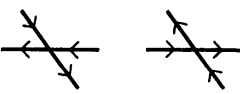

$-x_{2}$

$-x_{2}$
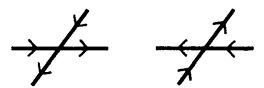

$-x_{3}$
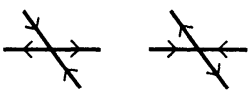

$1+x_{2} q^{-1}$

$1+x_{2} q$
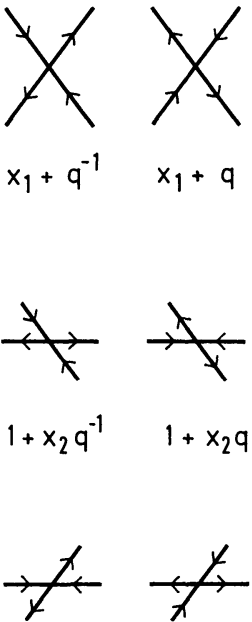

$x_{1}+q$

$1+x_{3} q^{-1} \quad 1+x_{3} q$

and matrices adding a vertex read as (41), where the Temperley Lieb representation is (12). Commutation of $\tau_{\text {vertex }}$ with $U_{q} s l(2)$ follows, where again

$$
Q^{1 / 2}=q+q^{-1} \text {. }
$$

Expressions $(20,23)$ still hold true.

Although $G$ is not self dual, a combination of duality and star triangle transformation exists that map $G$ onto itself $[30,31]$. The fixed critical line is given by

$$
Q^{1 / 2} x_{1} x_{2} x_{3}+x_{1} x_{2}+x_{1} x_{3}+x_{2} x_{3}=1
$$

The thermodynamic properties of the vertex model when (45) is satisfied have very simple expressions in terms of the same properties on the square lattice. For instance [2]

$$
f_{\text {vertex }}^{\mathrm{tri}}\left(x_{1} x_{2} x_{3}\right)=\frac{1}{3} \sum_{i=1}^{3} f_{\text {vertex }}^{\text {square }}\left(x_{i}\right)
$$

Moreover we expect the universality classes to be the same. For $x_{i} \geqq 0(45)$, this is the known ferromagnetic Potts model. For $x_{i} \leqq 0$ in (45), we should recover properties of Sect. 4. This is easily checked in the anisotropic limit with $x_{2}, x_{3} \rightarrow 0^{-}$ 


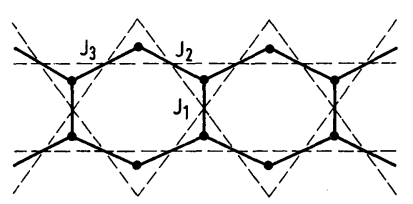

Fig. 3. The hexagonal lattice and its medial Kagomé lattice

while $x_{1} \rightarrow-\infty$. Then (45) implies $x_{2}+x_{3} \simeq \frac{1}{x_{1}}$, and

$$
\tau_{\mathrm{Potts}} \simeq x_{1}^{l}\left(1+\frac{1}{x_{1}} \sum_{i=1}^{2 l} e_{i}\right),
$$

so the hamiltonian is again (27).

In the isotropic case and for $x_{i} \leqq 0$, line (45) stops being physical at $Q=2$, and for $Q>2$, the corresponding coupling is imaginary.

Results for the honeycomb lattice can then be deduced by duality. The medial graph $G^{\prime}$ is also a Kagomé lattice (Fig. 3), and vertex weights are similar to (43), with $x_{i}^{\text {honeycomb }}=\frac{1}{x_{i}^{\text {triangular }}}$. The criticality condition reads thus

$$
\sqrt{Q}+x_{1}+x_{2}+x_{3}=x_{1} x_{2} x_{3} .
$$

Within the universality hypothesis, the $Q$ state Potts model on lines $(25,45,48)$ with negative $x_{i}$ 's should be critical at the Beraha values $Q=B_{n}$. Consequently we expect real zeros of $\mathscr{Z}_{G}(Q, x)$ to converge to all $B_{n}$ 's for $t \rightarrow \infty$ and $l$ large enough, including $t, l \rightarrow \infty$.

\section{Zeroes of the Chromatic Polynomial for the Square Lattice}

At the present stage we have shown that in the Potts model parameter space there is a line

$$
e^{K}=1-\sqrt{Q}
$$

along which $\mathscr{Z}_{G}\left(Q, e^{K}\right)$ has real zeros in the variable $Q$ which accumulate to the $B_{n}$ 's (and only to these) in the thermodynamic limit. The discussion of the chromatic polynomial $P_{G}=\mathscr{Z}_{G}(Q, 0)$ is more difficult.

On the one hand we still have at our disposal the algebraic structure of Sects. 2,3 , so formulas $(20,23)$ still hold true. But we do not have much information concerning the eigenvalues $\lambda_{j}^{\alpha}$ themselves. Since the argumentation leading to zeros of $\mathscr{Z}_{G}$ at the $B_{n}$ 's relied on both the $U_{q} s l(2)$ representation theory and the functional dependence (30), we cannot be so precise about $P_{G}$. However a discussion is still possible under some additional assumptions.

We noticed that line (49) is critical. To reach the zero temperature antiferro line

$$
e^{K}=0
$$

we have to describe some path in the Potts model parameter space. What makes sense physically is to tune the coupling $e^{K}$ to zero at fixed $Q$. Away from (49), the 


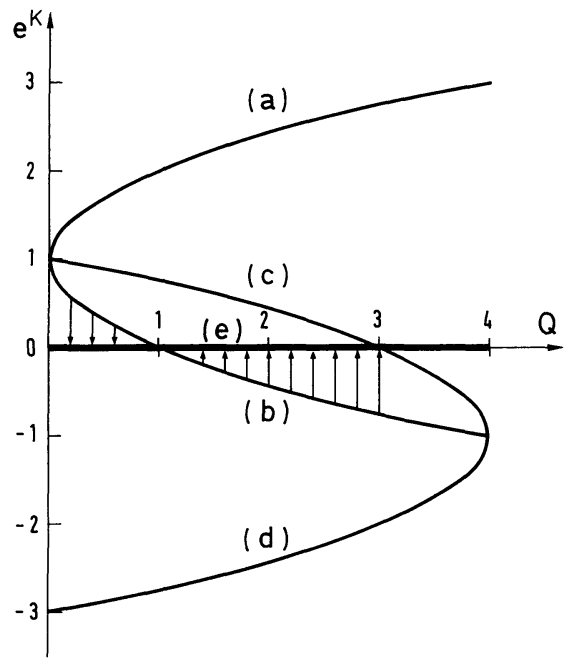

Fig. 4. Parameter space of the square lattice Potts model. Identified critical lines are: (a) $e^{K}=1$ $+Q^{1 / 2}$, (b) $e^{K}=1-Q^{1 / 2}$, (c) $e^{K}=-1+(4-Q)^{1 / 2}$ and its dual (d) $e^{K}=-1-(4-Q)^{1 / 2}$, (e) is the line $e^{K}=0$ giving the chromatic polynomial, (e) can be reached from (b) without crossing (c) provided $Q \leqq 3$

Potts model and the vertex model should become massive, and finite gaps appear. For $l$ finite, provided the approach to (49) is regular, the few largest eigenvalues should keep the same structure as in Sect. 4, with gaps (going as $\frac{1}{l}$ in case (49)) widening, while coincidences at $q=e^{i \pi m / n}$ still must occur due to $U_{q} s l(2)$ symmetry.

Hence the argumentation of Sects. 5, 6 leading to real zeros accumulating at the $B_{n}$ 's should extend away from line (49), until we reach some other critical point where the structure of the spectrum may be modified and some eigenvalues cross. In the $\left(Q, e^{K}\right)$ plane, Baxter has [32] exhibited an antiferromagnetic critical line (Fig. 4)

$$
e^{K}=-1+(4-Q)^{1 / 2} \text {. }
$$

By turning $e^{K}$, we can reach line (50) from (49) without crossing (51) provided $Q \leqq 3$. In which case, assuming lines abcd of Fig. 4 are the only critical lines in the region of interest, the spectrum structure of Sect. 4 should be correct in the $e^{K}=0$ case as well.

When $Q>3$, we cannot reach (50) from (49) without crossing (51), and the results of Sect. 4 cannot be extended without further analysis. A study of line (51) and the vicinity of $Q=3$ would be interesting by itself, and we hope to report on it later. For our purpose, we simply report here a numerical study of the staggered 6 vertex model for $e^{K}=0$. Some results are shown Figs. 5, 6 for $l=3$. We see that provided $\delta=6$, there occur numerous crossings, and for $\delta>6$, the structure of eigenvalues has been deeply changed, the largest one always occurring at $J=1$ independently of $\delta$. Other calculations show similar features occurring when (51) is crossed. (We found numerically $J=0$ for $l$ even, $J=1$ for $l$ odd.) 


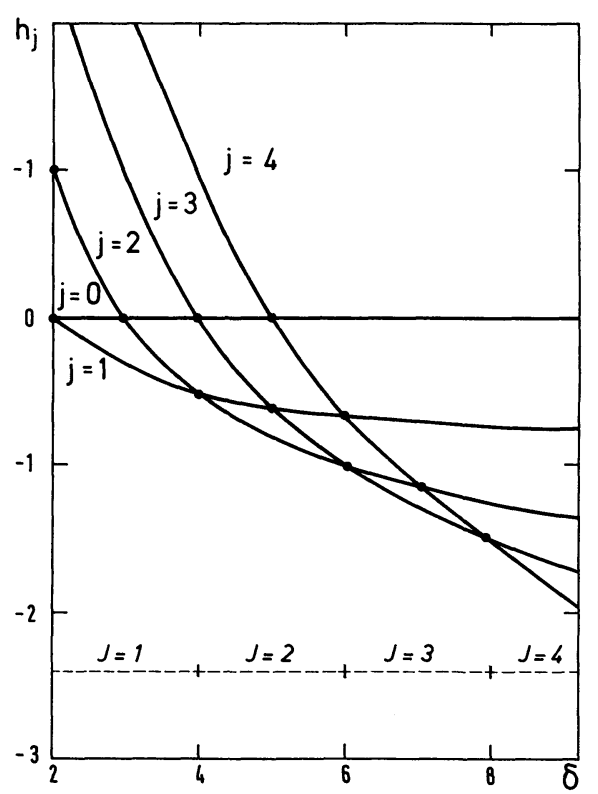

Fig. 5

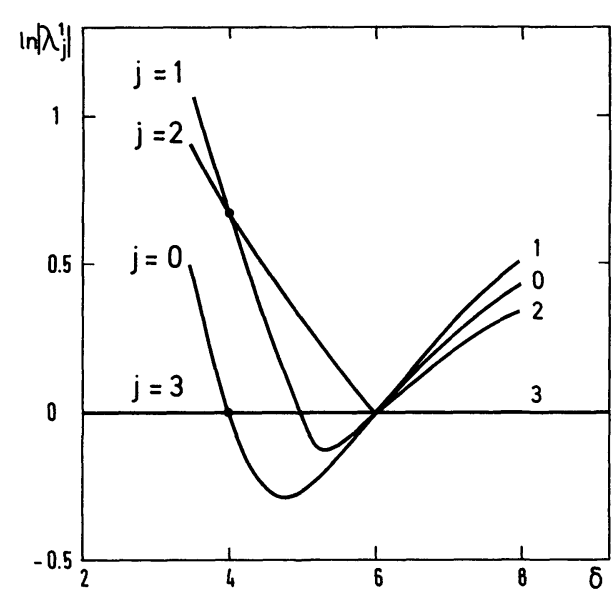

Fig. 6

Fig. 5. Dependence of $h_{j}(30)$ upon $\delta$. Crossing (indicated by dots) are due to $U_{q} s l(2)$ structure

Fig. 6. Numerical values of $\ln \left|\lambda_{j}^{1}\right|$ for $l=3$ as a function $\delta$ in the chromatic polynomial case. For $\delta \leqq 6$ the structure is qualitatively the same as Fig. 5. For $\delta>6$ the ground state is $\lambda_{1}^{1}, J=1$. Notice the very large degeneracy at $\delta=6$

Since for $Q>3, J \leqq 1$ on (50), the largest eigenvalues of the Potts and vertex models are now identical, and there is no singularity left at the $B_{n}$ 's.

Hence we expect real zeros of $P_{G}$ converging to $B_{1} \ldots B_{5}$ only (and maybe $B_{6}$. This point is at the edge of our reasoning and we are not sure about its status), and only, and no zeros after $Q=3$. So far, neither $B_{5}$ nor $B_{6}$ have been observed numerically.

\section{Zeros of the Chromatic Polynomial. Triangular and Honeycomb Lattices}

The reasoning is in principle the same for these other lattices. In the triangular case, it is supposed [32] that the antiferro critical line coincides with the zero temperature one. Thus when turning $e^{K}$ to zero from (45) at fixed $Q$, a critical point is always met. Fortunately some additional information is known from Baxter's work [10]. A crossing of eigenvalues is localized at $B_{14}<Q_{0} \simeq 3.8197<B_{15}$ (this point may be part of some other critical line in Fig. 7). By analogy with the square lattice case, the structure of eigenvalues of Sect. 4 should extend up to $\delta \lesssim 14$ on line (50), with the usual consequence, while for $\delta \gtrsim 15$ it should exhibit a maximum at $J \leqq 1$. Hence we expect real zeros of $P_{G}$ converging to $B_{1} \ldots B_{14}$.

Finally for the honeycomb case things are more mysterious. The image of the antiferro critical line of the triangular lattice under duality is

$$
e^{K}=1-Q \text {. }
$$




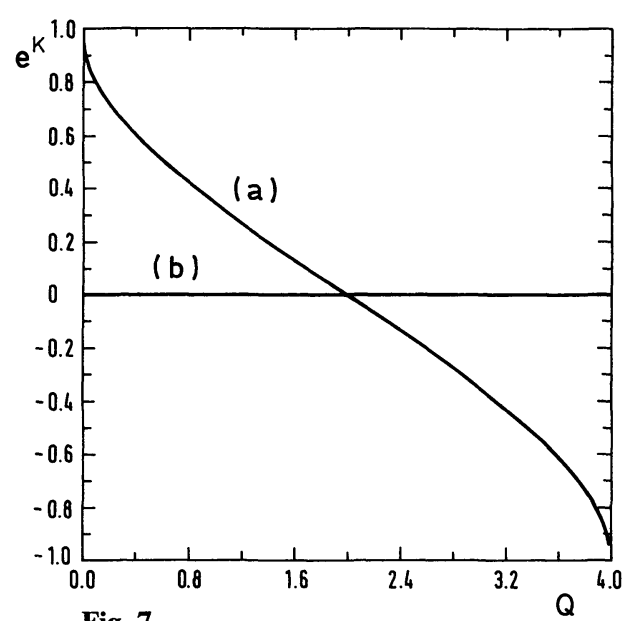

Fig. 7

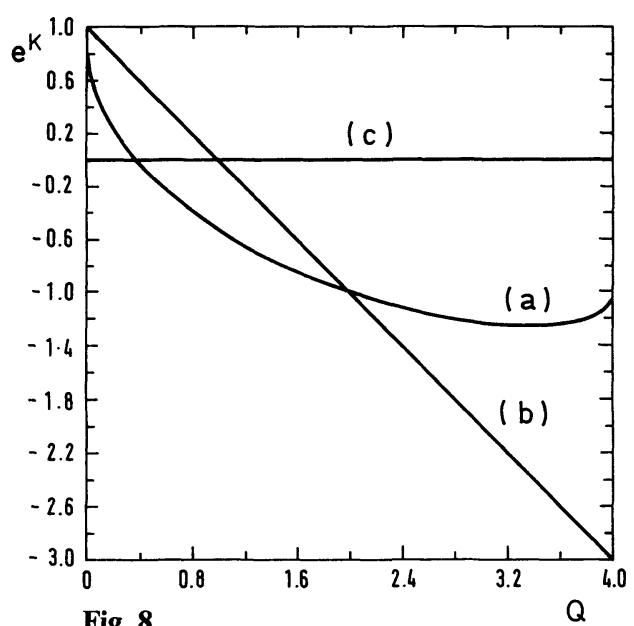

Fig. 8

Fig. 7. Parameter space of the triangular lattice Potts model. Known critical lines are: (a) $Q^{1 / 2} x^{3}$ $+3 x^{2}=1$, (b) $e^{K}=0$. On (b), a crossing is identified at $Q_{0} \simeq 3.8197$

Fig. 8. Parameter space of the honeycomb lattice Potts model. Known critical lines are: (a) $Q^{1 / 2}$ $+3 x=x^{3}$, (b) $e^{K}=1-Q$. The chromatic polynomial line is (c) $e^{K}=0$. It can be reached from (a) without crossing (b) for $0 \leqq Q \leqq 1$ or $Q \geqq 2$

Tuning $e^{K}$ to 0 from (48), we do not cross a known critical line for $Q<1$ or $Q>2$, and cross (52) for $1<Q<2$. It seems quite unlikely that the eigenvalues structure changes twice along (52). Rather, there is probably some other singularity away from (48) for $Q \geqq 2$ which forbids continuing results up to (50). We expect real zeros to occur and converge to $B_{2} \ldots B_{4}$ only. This seems confirmed by numerical results [10].

\section{More Physical Questions}

We want to discuss here some physical consequences or interpretations of our results.

a) First we comment an $f_{\text {Potts }}$ and $f_{\text {vertex }}$ on line (49) and $Q=B_{n}$. When looking at $\chi_{j}^{(l, t)}(24)$ we keep only $\Omega_{j}^{2 l}$ levels among the $\Gamma_{j}^{2 l}$ initial ones. As is well known, $\Omega_{j}^{2 l}$ is also the number of configurations of an $A_{n-1}$ model on a strip of width $l$, with height on the top (upper) row fixed to $1(1+2 j)[21]$. Hence

$$
\Omega_{j}^{2 l}=\left\langle 1\left|\left(C_{A_{n-1}}\right)^{l}\right| 1+2 j\right\rangle,
$$

where $C_{A}$ is the incidence matrix of the $A_{n-1}$ Dynkin diagram. For $l$ large, (53) is dominated by the largest eigenvalue

$$
\Omega_{j}^{2 l} \sim\left(2 \cos \frac{\pi}{n}\right)^{l} .
$$

Due to the cancellations in (24), the $\Omega_{j}^{2 l}$ are in the most excited levels of the $\Gamma_{j}^{2 l}$ ones. Suppose they are the most excited ones and that moreover we can for finite $l$ 
replace each $\lambda$ by its asymptotic behaviour. Then the largest eigenvalue of the Potts model in the $j$ sector $\Lambda_{j}^{\text {Potts }}$ should scale like $\Lambda e^{-\pi h / l}$, where $h=h_{j}+k$, and the integer $k$ in such that

$$
\sum_{i \leqq k} p(i) \sim \Gamma_{j}^{2 l}-\Omega_{j}^{2 l} \sim \Gamma_{j}^{2 l} \sim 2^{2 l},
$$

where $p(i)$ is the number of partitions of the integer $i$, occurring due to the expansion of the denominator in (34). Using known results on the asymptotic behaviour of $p(i)$ [32],

$$
\sum_{i \leqq k} p(i) \simeq k p(k) \sim e^{\mathrm{cst} \sqrt{k}}
$$

we find

$$
k \sim l^{2}
$$

Hence

$$
\left(\Lambda_{j}^{\text {Potts }}\right)^{t} \simeq \Lambda^{t} e^{- \text {cstlt }} \simeq e^{\left(f^{\text {vertex }- \text { cst }) l t} .\right.}
$$

From this qualitative picture we see that the behaviour of $\chi_{j}^{(l, t)}$ due to the extensive (vertex) term diverging exponentially and the $K_{j}$ series vanishing exponentially has still an extensive (Potts) behaviour, with however a different power. Putting correct figures in the above equations is a difficult task, probably analogous to the analysis of [13] in regime II.

To summarize we have shown that for $\delta \notin \mathbb{N}$, the free energy of the Potts and vertex models in the strip limit $t \rightarrow \infty$ coincide $f_{\text {Potts }}^{l}=f_{\text {vertex }}^{l}$. For $\delta=n$, and $l$ large enough but finite (typically $\left.l \gtrsim \frac{n-1}{2}\right)$ the free energy of the Potts and vertex models are different

$$
f_{\text {Potts }}^{l} \neq f_{\text {vertex }}^{l} \text {. }
$$

Moreover the preceding argument shows that (59) holds true even if $l \rightarrow \infty$. For $\delta=n>3$ it is reasonable to expect $f_{\text {Potts }}=\lim _{l \rightarrow \infty} f_{\text {Potts }}^{l}$ to be given by the other solutions of inversion identities [33,34], namely

$$
\begin{aligned}
& f_{\text {Potts }}=-\int_{-\infty}^{\infty} \frac{\operatorname{sh} \gamma t \operatorname{sh}(2 u-\pi) t}{2 t \operatorname{sh} \pi t \operatorname{ch} \gamma t} d t \quad \frac{\pi}{2}<u<\gamma+\frac{\pi}{2}, \\
& f_{\text {Potts }}=-\int_{-\infty}^{\infty} \frac{\operatorname{sh}^{2} \gamma t \operatorname{sh}(2 \pi-2 u) t}{t \operatorname{sh} \pi t \operatorname{sh}(\pi-2 \gamma) t} d t \quad \gamma+\frac{\pi}{2}<u<\pi,
\end{aligned}
$$

while it is known that [35]

$$
f_{\text {vertex }}=\int_{-\infty}^{\infty} \frac{\operatorname{sh} \gamma t \operatorname{sh} 2(\pi-u) t}{2 t \operatorname{sh} \pi t \operatorname{ch}(\pi-\gamma) t} d t \quad \gamma<u<\pi .
$$

$f$ is negative in (60), consistent with the fact that the ground state of the Potts model occurs in the set of very excited vertex states. Results $(60,61)$ are obtained with normalisations

$$
\begin{aligned}
X_{2 i-1} & \equiv\left(1+\frac{1}{x_{1}} e_{2 i-1}\right)=\left(1+x e_{2 i-1}\right), \\
X_{2 i} & \equiv\left(1+x_{2} e_{2 i}\right)=\left(1+x e_{2 i}\right) .
\end{aligned}
$$


Although for finite $t, l, F_{\text {Potts }}(Q, x=-1)$ is regular, in the strip limit $t \rightarrow \infty$, and for $l$ finite $\gtrsim \frac{n-1}{2}$, the free energy per bond $f_{\text {Potts }}^{l}$ is discontinuous at Beraha numbers $Q=B_{n}$. This behaviour still holds in the two dimensional $l \rightarrow \infty$ limit. We discussed the case of geometry (Fig. 1) only, but expect that $f_{\text {Potts }}, f_{\text {vertex }}$ do not depend on the boundary conditions in the thermodynamic limit.

This analysis should clarify the questions of "Temperley Lieb equivalence" between the Potts and 6-vertex model raised in $[33,34]$. Due to the $U_{q} s l(2)$ symmetry, so many coincidences of levels can occur that $f_{\text {Potts }} \neq f_{\text {vertex }}$, even if algebraically the correspondence (20) between the two models is the same as in the $x_{1}, x_{2}>0$ case. Our conclusions disagree slightly with Baxter in that the expressions (60) should apply only at Beraha values $Q=B_{n}, n>3$. In Ref. [33, 34] it was instead conjectured that (60) applied to all $Q \in] 1,4]$. Of course comparison with other results at $Q=2=B_{4}, Q=3=B_{6}$ was correct.

b) Second we notice that the vertex model with $u=\pi-\frac{\gamma}{2}, \gamma=\frac{\pi}{n}$ is related by the usual $[15,36]$ quantum group analysis to the $A_{n-1}$ RSOS model at the regime I-II transition. Although the continuum limit of the vertex model is described by only one free field, the continuum limit of the $A_{n-1}$ face model which is the $\mathbb{Z}_{n-2}$ universality class [37], involves several free fields depending on the picture [38]. The origin of this different description should be hidden in the mechanism of level cancellations. Indeed the analysis of Ref. [13] shows in the off-critical case how to obtain string functions from sums as (37). For RSOS models, the Bethe ansatz calculations of [39] showed that (60b) for $n>3$ is correct.

c) Third, via the polygon representation (19) and Euler's relation, $\mathscr{Z}_{G}$ reads for $x_{1}=x_{2}=-1$,

$$
\mathscr{Z}_{G}(Q, x=-1)=\left(-Q^{1 / 2}\right)^{l t} \sum_{\mathscr{G}^{\prime}}\left(-Q^{1 / 2}\right)^{P} .
$$

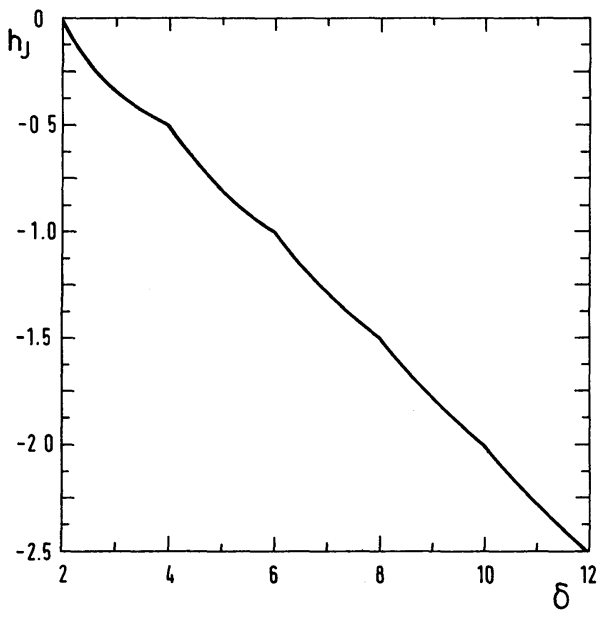

Fig. 9. $h_{J}$ (30) (32) as a function of $\delta$ is piecewise regular. For $2 n^{\prime}<\delta<2 n^{\prime}+2, J=n^{\prime}$, $h_{J}=\frac{n^{\prime 2}+n^{\prime}}{\delta}-n^{\prime}$. Jumps in derivatives occur at every even $\delta=2 n^{\prime}$ 
This is a "dense" loop model [40] with a weight $-Q^{1 / 2}$ per polygon. It would be nice to find a geometrical interpretation of singularities of (63) at the $B_{n}$ 's.

d) Finally some Beraha numbers also are special points for the vertex model. For instance the scaling behaviour of the vertex ground state is given by $h_{J}-\frac{c}{24} \cdot h_{J}(\delta)$ is made of pieces of curves (Fig. 9) with jumps in the derivatives at each $\delta=2 n^{\prime}$. The effective central charge (33) reaches its upper value $c_{\text {app }}=1$ at paints $\delta=2 n^{\prime}+1$.

\section{Conclusions}

To study the chromatic polynomials $P_{G}(Q)$, we have introduced a $Q$-state Potts model on $G$, and embedded the latter in a staggered 6-vertex model which exhibits $U_{q} s l(2)$ symmetry. For the square, triangular and honeycomb lattices and graphs $G_{l, t}$ as in Figs. 1-3, we have exhibited an antiferro like critical line along which the $Q$-state Potts model is singular, in the thermodynamic limit $l, t \rightarrow \infty$, at all the $B_{n}$ 's. Consequently, real zeros for finite systems occur depending on the sizes $l, t$ and accumulate exponentially fast at the $B_{n}$ 's as $l, t \rightarrow \infty$. The zero temperature antiferro line that corresponds to $P_{G}(Q)$ has then to be reached from the above critical line by turning $e^{K}$. The $U_{q} s l(2)$ symmetry and its peculiarities for $q$ a root of unity always hold true, but the eigenvalue structure may be destroyed if some singularity is encountered. Using known results on the parameter space of the $Q$-state Potts model, we argued that real zeros should occur, and accumulate to $B_{n}$ 's for $1<n \leqq n_{0}$ only, where $n_{0}$ depends on $G\left(n_{0}=6,14,4\right.$ for the square, triangular and honeycomb lattice).

We have moreover obtained information of the size dependence of the zeroes. For instance, in a strip geometry and $1<n \leqq n_{0}$, we expect real zeros to occur and accumulate exactly at $B_{n}$ when $t \rightarrow \infty$ and $l$ finite $\gtrsim \frac{n-1}{2}$.

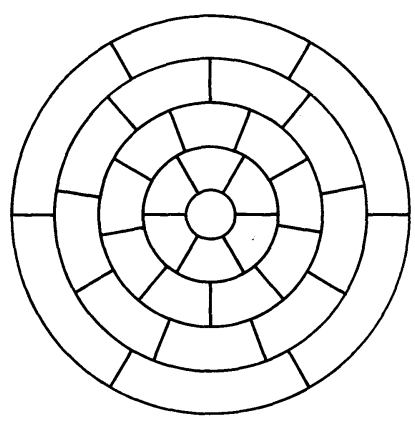

Fig. 10

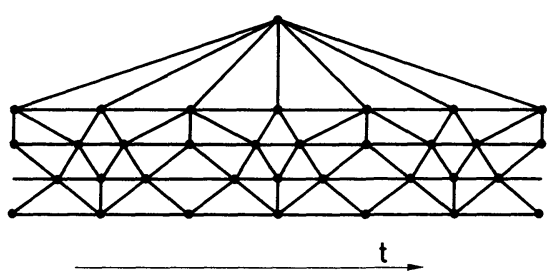

Fig. 11

Fig. 10. The authors of ref. [8] considered the chromatic polynomial of this graph, with the point of view of colouring neighbouring regions with different colour

Fig. 11. The dual of Fig. 10 where points are introduced in the middle of regions and edges between neighbouring regions, can be unfolded as Fig. 11, with periodic boundary conditions in time direction 
Table 4. The first real zeros of the chromatic polynomial of the graph Figs. 10 and 11 (from [8])

$$
\begin{aligned}
& x_{0}=0 \\
& x_{1}=1 \\
& x_{2}=2 \\
& x_{3}=2.61803399 \\
& x_{4}=3 \\
& x_{5}=3.2469919 \\
& x_{6}=3.41539930 \\
& x_{7}=3.52004593
\end{aligned}
$$

We do not consider our work as a full proof. Indeed, many steps look physically reasonable, but lack some rigour. Among them are the assumption that Bethe states are complete, that the asymptotic behaviour of eigenvalues obeys conformal invariance prediction, that the Potts model is regular except on some critical lines in the $\left(e^{K}, Q\right)$ parameter space. On the other hand, it seems that our analysis provides what may be for the first time a plausible explanation of Beraha's conjecture. It must be emphasized that an extension of the above arguments is partly possible, since the Potts model-vertex correspondence and the transfer matrix formalism can be generalized to other graphs. For instance the authors of [8] considered the chromatic polynomial of the graph $G$ in Fig. 10. They had the point of view of colouring regions such that neighbouring ones (i.e. with a common border) have different color. This is easily transferred into the problem we considered by turning to the dual lattice with points carrying Potts variables at the center of faces, and edges between neighbouring regions. We can then unfold this dual of Fig. 10 into the system of Fig. 11, where periodic boundary conditions are imposed in time direction. $P_{G}(Q)$ has been calculated in [8], with the first real zeros indicated Table 4 , very close to $B_{2} \ldots B_{9}$.

The Potts model on $G$ can also be embedded into a vertex model. First notice that the Potts partition function on $G$ can be written as

$$
\mathscr{Z}_{G}(Q)=\operatorname{Tr}\left(\tau_{\text {Potts }}\right)^{3} \text {, }
$$

where $\tau$ propagates on an elementary cell. Restricting to the case of isotropic interactions with

we have

$$
X_{2 i-1}=\left(x+e_{2 i-1}\right), \quad X_{2 i}=\left(1+x e_{2 i}\right)
$$

$$
\begin{aligned}
\tau_{\text {Potts }}= & X_{7} X_{8} X_{6} X_{9} X_{5} X_{8} X_{6} X_{4} X_{2} X_{7} X_{3} X_{6} X_{4} X_{5} \\
& \times X_{8} X_{6} X_{4} X_{2} X_{7} X_{3} X_{8} X_{6} X_{4} X_{9} X_{5} X_{8} X_{6} X_{4} X_{2} .
\end{aligned}
$$

Although this looks a bit complicated, the important point is that $\tau_{\text {Potts }}$ expands on Temperley Lieb matrices. Hence a vertex model on the medial graph can be introduced with the other representation (12), commutation with $U_{q} s l(2)$ and decomposition formula (20) still hold true. Provided the eigenvalue structure of Sect. 4 applies here, the mechanism producing zeros close to the $B_{n}$ 's for $G$ in Figs. 10 and 11 should be the same as before. For instance, if we considered $\mathscr{Z}_{G}=\operatorname{Tr} \tau_{\text {Potts }}^{l}$ for $l$ large, zeros corresponding to Table 4 should exactly converge to the $B_{n}$ 's. 


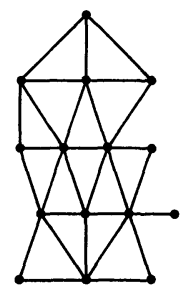

Fig. 12. Cell corresponding to propagation with $\tau_{\text {Potts }}(66)$

For a family of two dimensional graphs consisting of pieces of a two dimensional lattice (i.e. a Bravais lattice plus some arbitrary cell) wrapped on a cylinder as in Figs. 1-3, the scheme of our analysis should apply, making the Beraha conjecture as formulated in [2] at least reasonable. An important question we have not discussed is the effect of changing boundary conditions. It should be easy to switch from our cylindrical boundary conditions to free ones, and numerical results of [10] show indeed little difference between the two cases. More intriguing are toroidal boundary conditions. Although they usually do not affect the thermodynamic behaviour, the situation may be different in our case. It is known for instance that the four colors theorem becomes then a seven colors one [1].

Finally it is interesting to notice that Beraha numbers provide an example of rather strong universality of critical points, since the only thing that depends on the lattice is whether or not a given $B_{n}$ is a singularity point of the $Q$-state, zero temperature antiferromagnetic Potts model.

\section{References}

1. Saaty, T., Kainen, P.: The four color problem. New York: Mcisraw-Hill 1977 and references therein

2. Baxter, R.J.: Exactly solved models in statistical mechanics. New York: Academic Press 1982

3. Wu, F.Y.: Rev. Mod. Phys. 54, 235 (1982)

4. Beraha, S.: Infinite non-trivial families of maps and chromials, thesis, John Hopkins University

5. Beraha, S., Kahane, J., Weiss, N.: In: Studies in foundations and combinatorics, Adv. Math. Suppl. Stud. 1, 213 (1978)

6. Beraha, S., Kahane, J., Weiss, N.J.: J. Comb. Theory B 28, 52 (1980)

7. Tutte, W.T.: J. Comb. Theory 9, 289 (1970)

8. Tutte, W.T.: Can. J. Math. 25, 426 (1973)

Hall, D.W., Siry, J.W., Vanderslice, B.R.: Proc. Am. Math. Soc. 16, 620 (1965)

9. Biggs, N.L., Damerall, R.M., Sands, D.A.: J. Comb. Theory B 12, 123 (1962)

10. Baxter, R.J.: J. Phys. A 19, 2821 (1986); J. Phys. A 20, 5241 (1987)

11. Jones, V.: Invent. Math. 72, 1 (1983)

12. Friedan, D., Qiu, Z., Shenker, S.: Phys. Rev. Lett. 52, 1575 (1984)

13. Andrews, G.E., Baxter, R.J., Forrester, P.J.: J. Stat. Phys. 35, 193 (1983)

14. Pasquier, V.: Nucl. Phys. B 295, 491 (1988)

15. Pasquier, V., Saleur, H.: Nucl. Phys. B 330, 523 (1990) 
16. Alvarez Gaumé, L., Gomez, C., Sierra, G.: Phys. Lett. B 220, 142 (1989)

17. Gervais, J.L.: The quantum group structure of $2 \mathrm{D}$ gravity and minimal models. Preprint LPTENS 1989

18. Drinfeld, V.G.: Dokl. Akad. Nauk. SSSR 283, 1060 (1985)

Jimbo, M.: Lett. Math. Phys. 10, 63 (1985)

Drinfeld, V.G.: Proc. ICM (AMS Berkeley) 1978 and references therein

19. Baxter, R.J., Kelland, S.B., Wu, F.Y.: J. Phys. A 9, 397 (1976)

20. di Francesco, P., Saleur, H., Zuber, J.B.: J. Stat. Phys. 49, 57 (1987)

21. Bauer, M., Saleur, H.: Nucl. Phys. B 320, 591 (1989)

22. Lusztig, G.: Modular representations and quantum groups. Preprint 1989

23. Roche, P., Arnaudon, D.: Lett. Math. Phys. 17, 295 (1989)

24. Felder, G.: Nucl. Phys. B 317, 215 (1989)

25. Sklyanin, E.K.: Boundary conditions for integrable quantum systems. Preprint 1986

26. Gaudin, M.: La fonction d'onde de Bethe. Paris: Masson 1983

27. de Vega, H., Woynarovich, F.: Nucl. Phys. B 251, 439 (1985)

Hamer, C.J., Quispel, G.R.W., Batchelor, M.T.: J. Phys. A 20, 5677 (1987)

28. See Itzykson, C., Saleur, H., Zuber, J.B.: Conformal invariance and applications to statistical mechanics. Singapore: World Scientific 1988 and references therein

29. Kim, D., Pearce, P.A.: J. Phys. A 20, L 451 (1987)

30. Kim, D., Joseph, R.I.: J. Phys. C 7, L 167 (1974)

31. Baxter, R.J., Temperley, H.N.V., Ashley, S.E.: Proc. Roy. Soc. London A 358, 535 (1978)

32. Andrews, G.E.: Theory of partitions. Reading, MA: Addison Wesley 1976

33. Baxter, R.J.: J. Stat. Phys. 28, 1 (1982)

34. Baxter, R.J.: Proc. R. Soc. Lond. A 383, 43 (1982)

35. Lieb, E.H., Wu, F.Y.: In: Phase transitions and critical phenomena. Vol. 1, Domb, C., Green, M.S. (eds.). London 1972

36. Pasquier, V.: Commun. Math. Phys. 118, 355 (1988)

37. Zamolodchikov, A.B., Fateev, V.A.: Nucl. Phys. B 240, 115 (1984)

38. Distler, J., Qiu, Z.: BRS cohomology and a Feigin Fuchs representation of parafermionic theories. Preprint 1989

39. Bazhanov, V.V., Reshetikhin, N.Yu.: Int. J. Mod. Phys. A 4, 115 (1989)

40. Saleur, H.: Phys. Rev. B 35, 3657 (1987)

Duplantier, B., David, F.: J. Stat. Phys. 51, 327 (1988)

Communicated by K. Gawedzki

Received January 10, 1990

Notes added in proof. a) We have now a better understanding of the region between (49) and (50). It is a massless region (analogous to a Kadanoff Berker phase) with the same initical properties than (49). The conclusions of Sects. 4, 6 thus apply to the chromatic case, completing the argument of Sect. 8. b) We have generalized am argument to the case of free boundary condition also. 
